\title{
The Metabolism of $\boldsymbol{n}$-Decane by a Pseudomonas
}

\author{
BY C. W. BIRD AND P. MOLTON \\ Department of Chemistry, Queen Elizabeth College, London, W. 8
}

(Received 12 January 1967)

\begin{abstract}
The growth of a Pseudomonas on $n$-decane was found to produce stearic acid, oleic acid, palmitic acid, palmitoleic acid, decanoic acid, octanoic acid, $\beta$-hydroxydecanoic acid, $\beta$-hydroxyoctanoic acid, $\beta$-hydroxyhexanoic acid and $\beta$-hydroxyadipic acid. Small amounts of $n$-decanamide and $n$-valeramide were also isolated. The effects of nitrogen and oxygen limitation on the formation of these products in continuous fermentations is reported.
\end{abstract}

Though the ability of micro-organisms to utilize alkanes as their sole carbon source has been recognized for over 60 years, the metabolic pathway involved is as yet ill-defined (ZoBell, 1950; Van der Linden \& Thijsse, 1965). However, it is generally believed that the initial oxidative attack occurs at the terminal carbon atoms. The contrast with the low specificity observed in chemical attack on alkanes suggested the use of micro-organisms for the selective introduction of oxygen-containing functional groups at the terminal carbon atoms.

As a necessary preliminary to studies in this field we report the isolation and characterization of the normal metabolic products obtained from Pseudomonas $\mathrm{X} 2$, grown in continuous culture on a mineral-salt medium with $n$-decane as sole carbon source, and the influence of culture conditions on their formation.

\section{EXPERIMENTAL}

Isolation and growth of organism. The species of Pseudomonas, designated X2, was isolated from Thames Estuary mud by Professor S. J. Pirt by using a medium containing $n$-decane as the sole carbon source. Continuous fermentations were carried out by Mr D. G. MacLennan by using the first stage of the two-stage equipment described by Callow \& Pirt (1961), fitted with an automatic oxygentension control (MacLennan \& Pirt, 1967). A dilution rate of $0 \cdot 1 \mathrm{hr} .^{-1}$ at $30^{\circ}$, with the $\mathrm{pH}$ maintained at $7 \cdot 1$, gave a cell production of $1 \mathrm{~g}$. dry wt./l. In addition to $n$-decane (>99\% purity) the medium contained: $\mathrm{NaH}_{2} \mathrm{PO}_{4}(1.56 \mathrm{~g} . /$ l.), $\mathrm{K}_{2} \mathrm{HPO}_{4}\left(6.97 \mathrm{~g}\right.$./l.), $\mathrm{NH}_{4} \mathrm{Cl}\left(2 \cdot 0 \mathrm{~g}\right.$. $/ \mathrm{l}$.), $\mathrm{CaCl}_{2}(0.01 \mathrm{~g}$./1.), $\mathrm{Na}_{2} \mathrm{~B}_{4} \mathrm{O}_{7}, 10 \mathrm{H}_{2} \mathrm{O} \quad(0.1 \mathrm{mg} . / \mathrm{l}),. \mathrm{MgSO}_{4}, 7 \mathrm{H}_{2} \mathrm{O} \quad(0 \cdot 2 \mathrm{~g} . / 1$.$) ,$ $\mathrm{FeSO}_{4}, 7 \mathrm{H}_{2} \mathrm{O} \quad\left(0.5 \mathrm{mg}\right.$./1.), $\mathrm{MnSO}_{4}, 6 \mathrm{H}_{2} \mathrm{O} \quad(0.5 \mathrm{mg} . / 1$.$) ,$ $\mathrm{CuSO}_{4}, 5 \mathrm{H}_{2} \mathrm{O}\left(0 \cdot 1 \mathrm{mg}\right.$./l.), $\mathrm{Na}_{2} \mathrm{MoO}_{4}, 2 \mathrm{H}_{2} \mathrm{O}(0 \cdot 1 \mathrm{mg}$. $/ 1$.) and EDTA (disodium salt) $(0 \cdot 26 \mathrm{~g} . / 1$.$) .$

Chromatography. Analytical gas-liquid chromatography was carried out on a Pye Argon Chromatograph at $100^{\circ}$ and $150^{\circ}$ with a column packing of $10 \%$ polyethylene glycol adipate 1500 on 100-120-mesh acid-washed Celite. An Aerograph Autoprep, equipped with a $10 \mathrm{ft}$. $10 \%$ silicone
SE-30 on $80-100$-mesh Celite column and programmed from $80^{\circ}$ to $250^{\circ}$, was used for preparative gas-liquid chromatography.

Thin-layer chromatography was carried out on Merck silica gel $G$ with as solvent system either $27 \%(v / v)$ ether in light petroleum (b.p. $40-60^{\circ}$ ) or, for very polar material, chloroform. The components were detected by exposure to iodine vapour.

Spectra. Nuclear-magnetic-resonance (n.m.r.) spectra were recorded on a Varian HA-100 instrument for $\mathrm{CCl}_{4}$ solutions with tetramethylsilane as the internal standard. Mass spectra were measured on an A.E.I. M.S.9 instrument.

Isolation of extracellular products. The fermentation liquors (about 1901.) were collected from continuous cultures of Pseudomonas X 2 grown with various $n$-decane concentrations. The cells were removed either by continuous centrifugation or, more conveniently, by addition of sodium CM-cellulose ( $0 \cdot 6 \mathrm{~g}$./1.). A portion of the liquor was extracted at $\mathrm{pH} 7$ with ether. After removal of the ether, the resulting extract was chromatographed on silica gel. $n$-Decane was eluted with light petroleum (b.p. 40-60 and the remaining compounds were eluted with methanol. Separation by preparative gas-liquid chromatography yielded $n$-decanamide and $n$-valeramide.

The total fermentation liquor (1901.) was acidified to pH 2 with $\mathrm{H}_{2} \mathrm{SO}_{4}$ and extracted with dichloromethane. After drying over $\mathrm{Na}_{2} \mathrm{SO}_{4}$ and distilling off the dichloromethane, the residue was separated as above into $n$-decane $(560$ g.) and a brown viscous oil $(19 \cdot 3 \mathrm{~g}$.). After methylation with diazomethane, the oil was separated by preparative thin-layer chromatography into five fractions.

Fraction 1, $R_{F} 0 \cdot 77$ (5.7g.), was separated by preparative gas-liquid chromatography into seven further fractions, $1 a-1 g$. Fractions $1 a-1 c$ comprised only minor amounts of material of which the major component was identified by gas-liquid chromatography as methyl octanoate. Fraction $1 d(20 \mathrm{mg}$.) was methyl decanoate. At least ten components were present in fraction $1 e(5 \mathrm{mg}$.), but a mass spectrum showed no $m / e$ peaks at 214 and 242 indicative of methyl laurate and methyl myristate respectively. Fraction $1 f$ $(0 \cdot 25 \mathrm{~g}$.) was a mixture of methyl palmitate and methyl palmitoleate. A 1:1 composition was indicated both by gas-liquid chromatography and an n.m.r. spectrum that showed one olefinic proton $(\tau 4 \cdot 68)$ for every three methoxyl 
protons $(\tau 6 \cdot 40)$. The two esters were separated by thinlayer chromatography on silica gel made up with $0.1 \mathrm{~N}-$ $\mathrm{AgNO}_{3}$. Fraction $1 \mathrm{~g}(0.53 \mathrm{~g}$.) was a mixture of methyl stearate and methyl oleate. The n.m.r. spectra showed this to be a 1:3 mixture and this was separated into its components by thin-layer chromatography on $\mathrm{AgNO}_{3}$ impregnated silica gel.

Fraction $2, R_{F} 0 \cdot 25$ (3.1 g.), was separated by preparative gas-liquid chromatography into the two major components, methyl $\beta$-hydroxyoctanoate and methyl $\beta$ hydroxydecanoate.

Fraction $3, R_{F} 0 \cdot 18\left(0 \cdot 14 \mathrm{~g}\right.$.), and fraction $4, R_{F} 0 \cdot 12$ $(0.60 \mathrm{~g}$.$) , contained too many components for effective$ separation.

Fraction $5, R_{F} 0(0 \cdot 18 \mathrm{~g}$.$) , on preparative gas-liquid$ chromatography, yielded dimethyl $\beta$-hydroxyadipate (about $1 \mathrm{mg}$.). A portion (81.) of the acidified fermentation liquor, which had already been extracted with dichloromethane, was continuously extracted with ether for a week, giving an orange oil (1/1g.). After methylation with diazomethane, the oil was further separated by thin-layer chromatography, with $5 \%(\mathrm{v} / \mathrm{v})$ methanol in $\mathrm{CHCl}_{3}$, into three fractions with $R_{F}$ values $0-0.1$ (20mg.), $0 \cdot 1-0 \cdot 2$ (20 mg.) and 0.6-1.0 (1.0 g.). The last fraction was separated by preparative gas-liquid chromatography into methyl $\beta$-hydroxyhexanoate (20mg.) and methyl $\beta$-hydroxyoctanoate (50 mg.).

\section{Characterization and identification of products}

n-Decanamide. Retention time was identical with that of an authentic specimen. The mass spectrum shows peaks at $m / e 171(2 \cdot 9), 113(1), 112(1 \cdot 4), 97(5 \cdot 2), 83(6.9), 73(9)$, $72(40), 69(9 \cdot 9), 59(100), 55(16 \cdot 5), 44(14 \cdot 8)$ and $41(22 \cdot 6)$.

n-Valeramide. Retention time was identical with that of an authentic specimen. The mass spectrum shows peaks at $m / e ~ 101(1.5), 99(2 \cdot 2), 83(7 \cdot 4), 73(12 \cdot 4), 72(19 \cdot 5), 69$ $(9 \cdot 9), 59(100), 57(20), 55(21 \cdot 1), 44(16)$ and $41(28 \cdot 1)$.

Methyl decanoate. The retention time and i.r. spectrum were identical with published data. The mass spectrum shows peaks at $m / e 186,155,143,129,101,87$ and 74 (base peak). (Found: $\mathrm{C}, 70 \cdot 6 ; \mathrm{H}, \mathbf{1 1 \cdot 7}$. Calc. for $\mathrm{C}_{11} \mathrm{H}_{22} \mathrm{O}_{2}$ : $\mathrm{C}$, $71.0 ; \mathrm{H}, 11.8 \%$ ).

Methyl palmitate. The retention time and mass spectrum, $m / e 270$ and 74 (base peak), were identical with those of an authentic specimen.

Methyl palmitoleate. The retention time and mass spectrum, $m / e 268$ and 74 (base peak), were identical with those of an authentic specimen.

Methyl oleate. The retention time and mass spectrum, $m / e 296$ and 74 (base peak), were identical with those of an authentic specimen.

Methyl stearate. The retention time and mass spectrum, $m / e 298$ and 74 (base peak), were identical with those of an authentic specimen.

Methyl $\beta$-hydroxydecanoate. This had $[\alpha]_{\mathrm{D}}^{18}-16 \cdot 6 \pm 1^{\circ}$ (c 0.98 in $\mathrm{CHCl}_{3}$ ) (literature $[\alpha]_{\mathrm{D}}^{20}-18.3 \pm 1^{\circ}$; Cartwright, 1957) (Found: $\mathrm{C}, 65 \cdot 1 ; \mathrm{H}, 11 \cdot 1$. Calc. for $\mathrm{C}_{11} \mathrm{H}_{22} \mathrm{O}_{3}: \mathrm{C}$, $65 \cdot 3 ; \mathrm{H}, 11 \cdot 0 \%$ ). The n.m.r. spectrum showed (numbers of protons in parentheses): $\tau 6 \cdot 18$ (1) multiplet, 6.41 (3), 6.98 (1), 7.65 (1), 7.73 (1) doublet, 8.73 (12) multiplet and $9 \cdot 12$ (3) triplet. The mass spectrum shows peaks at $m / e$ $202,201,184,171,153,152,129,128,127,103$ (base peak), 74 and 71.
Methyl $\beta$-hydroxyoctanoate. This had $[\alpha]_{D}^{18}-18.5 \pm 1^{\circ}$ (c 1.0 in $\mathrm{CHCl}_{3}$ ) (literature $[\alpha]_{\mathrm{D}}-24^{\circ}$; Lemieux \& Giguere, 1951) (Found: $\mathrm{C}, 62 \cdot 0 ; \mathrm{H}, 10 \cdot 5$. Calc. for $\mathrm{C}_{9} \mathrm{H}_{18} \mathrm{O}_{3}: \mathrm{C}, 62 \cdot 1$; H, 10.4\%). The n.m.r spectrum showed (numbers of protons in parentheses) $\tau 6 \cdot 13$ (1) multiplet, $6 \cdot 38(3), 7 \cdot 00$ (1), 7.67 (1), 7.71 (2) doublet, 8.65 (8) multiplet and $9 \cdot 11$ (3) triplet. The mass spectrum shows peaks at $m / e 174$, $173,156,143,125,124,103$ (base peak), 74 and 71.

Methyl $\beta$-hydroxyhexanoate. The thin-layer-chromatographic $R_{F}$ value was characteristic of hydroxy ester. The mass spectrum shows peaks at $m / e 146(0.2), 145(1.5)$, $128(1 \cdot 7), 125(1 \cdot 8), 124(0.9), 117(2 \cdot 9), 116(3 \cdot 6), 115(43)$, $103(64), 74(46), 71(55), 61(29), 59(40), 55(41), 44(15)$, 43 (100), $42(15), 41(29), 40(3), 39$ (15), 31 (17) and $29(19)$. Dimethyl $\beta$-hydroxyadipate. The mass spectrum shows peaks at $m / e 191(0 \cdot 3), 171(33), 170(19), 149(24), 139(20)$, 126 (22), 112 (19), 87 (44), 74 (72), 69 (37), 59 (21), 57 (69), $56(25), 55(65), 43(59), 41$ (100), $39(26), 29$ (74) and $27(27)$. The gas-liquid-chromatographic retention time is different from that of dimethyl $\beta$-hydroxy- $\beta$-methylglutarate.

\section{RESULTS}

In view of the dangers inherent in relying solely on chromatographic techniques for identifying the metabolic products of Pseudomonas X 2, all of those present in adequate amounts in the culture medium have been isolated and further characterized.

In addition to stearic acid, oleic acid, palmitic acid, palmitoleic acid and decanoic acid, which were all isolated as their methyl esters, small amounts of octanoic acid were detected by gasliquid chromatography in the appropriate fraction. However, no evidence could be obtained for the presence of lauric acid and myristic acid.

Gas-liquid-chromatographic examination of the crude extracts indicated the presence of small amounts of methyl esters in the fermentation medium. $n$-Decanamide and $n$-valeramide were isolated and identified by their mass spectra and gas-liquid-chromatographic retention times. The latter criterion is particularly important in this case as the parent mass peaks of amides are notoriously difficult to identify, although the characteristic ions, $m / e$ 59, $\mathrm{CH}_{2}: \mathrm{C}(\mathrm{OH}) \cdot \stackrel{+}{\mathrm{NH}_{2}}$ and $m / e$ 44, $\stackrel{+}{\mathrm{C}} \mathrm{O} \cdot \mathrm{NH}_{2}$, are readily observed.

Three $\beta$-hydroxy acids, namely $D-\beta$-hydroxydecanoic acid, $D$ - $\beta$-hydroxyoctanoic acid and $\beta$-hydroxyhexanoic acid, were isolated also as their methyl esters. Their structures follow from their mass spectra (e.g. Fig. 1). The weak parent peak and strong $(M-1)^{+}$peak is charactoristic of hydroxy compounds as is also the formation of ions (M-18) corresponding to loss of water. The methyl ester grouping is indicated by the ion $m / e \mathbf{7 4}$, $\mathrm{CH}_{2}: \mathrm{C}(\mathrm{OH}) \cdot \mathrm{O} \cdot \stackrel{+}{\mathrm{C}} \mathrm{H}_{3}$, and this in conjunction with $+$

the ion $m / e$ 103, $\mathrm{HO}: \mathrm{CH} \cdot \mathrm{CH}_{2} \cdot \mathrm{CO} \cdot \mathrm{O} \cdot \mathrm{CH}_{3}$, places the hydroxyl group at the $\beta$-position. This assign- 


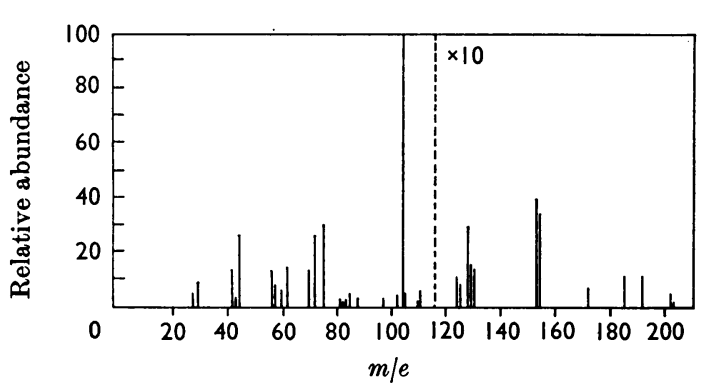

Fig. 1. Mass spectrum of methyl $\beta$-hydroxydecanoate.
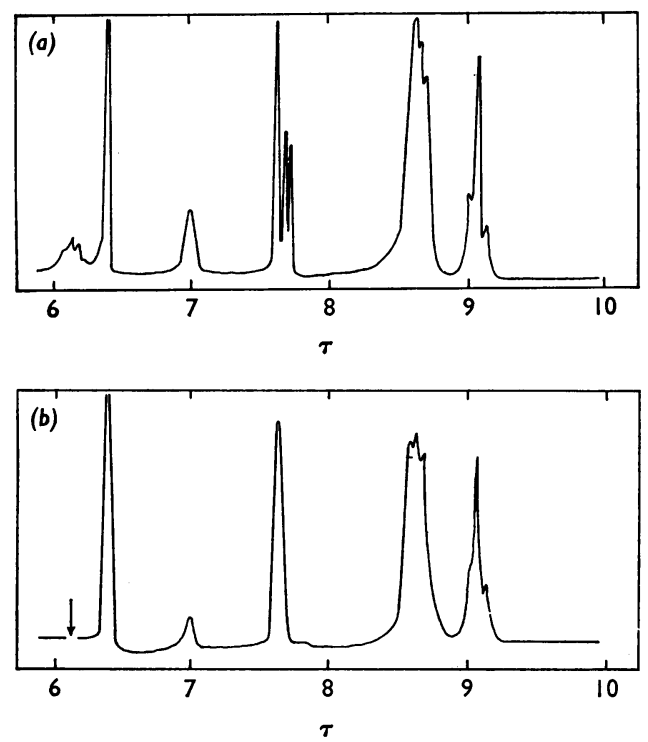

Fig. 2. (a) Nuclear-magnetic-resonance spectrum of methyl $\beta$-hydroxydecanoate at $100 \mathrm{Mcyc}$./sec. (b) Spectrum of methyl $\beta$-hydroxydecanoate when irradiated at the frequency indicated by arrow.

ment is further supported by the n.m.r. spectrum (Fig. 2a), which, in addition to absorptions at $\tau 6 \cdot 13$ $(\mathrm{O}-\mathrm{H}), 6.38\left(\mathrm{CH}_{3}-\mathrm{O}\right), 7.00(\mathrm{C}-\mathrm{H}), 8.65\left(\left[\mathrm{CH}_{2}\right]_{n}\right)$ and $9 \cdot 11\left(\mathrm{R}-\mathrm{CH}_{3}\right)$, shows a singlet (7.67) and doublet (7.71) attributable to a methylene adjacent to the carbonyl group and unsymmetrically coupled with the tertiary C-H group $(\tau 6 \cdot 13)$. On irradiation at this frequency the methylene bands collapsed to a singlet (Fig. 2b). As only minute quantities of dimethyl $\beta$-hydroxyadipate were obtained, its characterization rests on the mass spectrum, which shows the expected features (see above). The alternative identification as dimethyl $\beta$-hydroxy$\beta$-methylglutarate is excluded by a comparison of the gas-liquid-chromatography retention times with an authentic sample.
The effect of varying the growth conditions was examined to see whether intermediary primary metabolites could be accumulated. The results relevant to the present work are recorded in Table 1. The values quoted are the areas of the gasliquid-chromatographic peaks of the individual compounds relative to methyl $\beta$-hydroxydecanoate, and indicate how the relative proportions of products are affected by fermentation conditions. Comparison of columns 1 and 2 in Table 1 shows that increasing the concentration of $n$-decane results in a greatly increased production of fatty acids relative to hydroxy acids.

The experiments with oxygen limitation were carried out with the amount of ammonium chloride supplied adjusted so as to cause a $25 \%$ inhibition of cell growth at the highest oxygen partial pressure with a $2 \% n$-decane concentration. This ensured that $n$-decane was always present in excess. No appreciable qualitative changes occurred in the products formed. Comparison of columns 2 and 3 in Table 1 shows that nitrogen limitation causes a very marked decrease in the proportions of $\mathrm{C}_{16}$ and $\mathrm{C}_{18}$ acids and an increase in the amounts of $\mathrm{C}_{8}$ and $\mathrm{C}_{10}$ acids. Lowering the oxygen partial pressure results in a general decrease in acid formation with the exception of $\beta$-hydroxy acids. A changeover from nitrogen to oxygen limitation of growth occurs at an oxygen partial pressure between 5.5 and $3 \mathrm{~mm} . \mathrm{Hg}$, and is accompanied by a modest increase in fatty acid production.

\section{DISCUSSION}

The production of stearic acid, oleic acid, palmitic acid and palmitoleic acid by Pseudomonas $\mathrm{X} 2$ is unexceptional, although previous workers have not noted their formation. The presence of these acids indicates the operation of normal fatty acid synthesis in this organism. As the lower acids with even numbers of carbon atoms are presumably their precursors, all of them would be expected to be present, and hence the absence of lauric acid and myristic acid is surprising. This, and the presence of decanoic acid and octanoic acid, accompanied by the corresponding $\beta$-hydroxy acids, would appear at first sight to support the currently accepted metakolic pathway via terminal oxidation of the alkane ( $n$-decane) to the carboxylic acid, followed by successive $\beta$-oxidations. However, as the same products are obtained when the organism is grown on $n$-nonane such deductions are ill-founded. In addition, the $D$-configurations of the $\beta$-hydroxy acids are in better accord with a synthetic origin. It therefore seems unlikely that $\beta$-hydroxyadipic acid is derived from a diterminal oxidative degradation.

We have been unable to determine whether any 
Table 1. Effect of growth conditions on product formation by Pseudomonas X2

Bacteria were grown in defined medium (see the Experimental section), which was modified in the oxygen limitation experiments by adjusting the $\mathrm{NH}_{4} \mathrm{Cl}$ concentration so as to cause a $25 \%$ decrease in cell production at $29 \cdot 2 \mathrm{~mm}$. Hg oxygen partial pressure, with a $2 \% n$-decane concentration. The proportions of products relative to $\beta$-hydroxydecanoate are based on gas-liquid-chromatographic peak areas.

Proportions of products relative to $\beta$-hydroxydecanoate

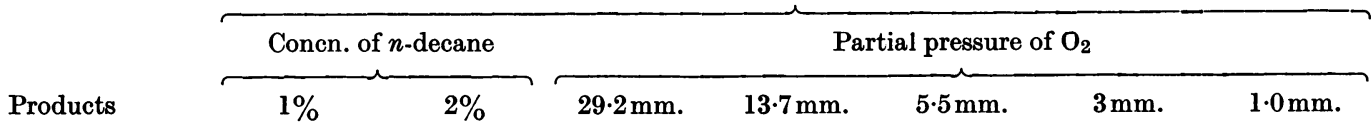

Dichloromethane extract

(g. of products/l. of medium supernatant)......

Octanoate

$0 \cdot 73$

$0 \cdot 56$

Decanoate

Palmitate + palmitoleate

Stearate+ oleate

$\beta$-Hydroxyoctanoate

$\beta$-Hydroxydecanoate

$4 \cdot 4$

102

120

$23 \cdot 3$

100

$1 \cdot 3$
$0 \cdot 37$
$8 \cdot 5$
570
460
37
100

$0 \cdot 93$
$1 \cdot 91$
$3 \cdot 41$
$27 \cdot 9$
$24 \cdot 6$
$79 \cdot 4$
100

$0 \cdot 13$
$1 \cdot 24$
$7 \cdot 96$
$7 \cdot 01$
$3 \cdot 72$
$73 \cdot 0$
100

$0 \cdot 13$
$0 \cdot 72$
$4 \cdot 28$
$4 \cdot 87$
$3 \cdot 35$
$65 \cdot 8$
100

$0 \cdot 30$
$1 \cdot 70$
$1 \cdot 93$
$9 \cdot 01$
$3 \cdot 60$
$62 \cdot 1$
100

$0 \cdot 17$

$1 \cdot 13$

$9 \cdot 01$

$10 \cdot 9$

$11 \cdot 3$

$63 \cdot 0$

100

of these compounds are formed by both degradative and synthetic pathways. The initial increase in the proportions of $\mathrm{C}_{10}$ and $\mathrm{C}_{8}$ acids observed in fermentations conducted under nitrogen limitation conditions (cf. columns 2 and 3 in Table 1), when the amounts of $\mathrm{C}_{16}$ and $\mathrm{C}_{18}$ acids have fallen sharply, would support a contribution from a degradative pathway. It is noteworthy that Thijsse \& Van der Linden (1963) found that chloramphenicol, which also limits cell growth, promotes the accumulation of propionic acid by a Pseudomonas growing on $n$-heptane.

The possible presence of hydroxy acids, which subsequently lactonize, in the fermentation liquors of another Pseudomonas sp. utilizing $n$-heptane has been noted (Ali Khan, Hall \& Robinson, 1964), and another isolate growing on $n$-hexadecane accumulates $\beta$-hydroxymyristic acid and $\beta$-hydroxylauric acid (Romero \& Brenner, 1966). In neither case was it established whether these were degradative or synthetic products, although a strain of Pseudomonas growing on glycerol forms glycolipids containing $\mathrm{D}$ - $\beta$-hydroxydecanoic acid (Bergström, Theorell \& Davide, 1947; Jarvis \& Johnson, 1949). The isolation of $\beta$-hydroxyadipic acid from natural sources has not been reported previously, whereas $\beta$-oxoadipic acid is a well-known intermediate in the biological degradation of aromatic hydrocarbons.

A further noteworthy feature is the formation of $n$-decanamide and $n$-valeramide. Small amounts of methyl esters of the accumulated acids have been detected by gas-liquid chromatography in the original extracts. Since we have found that the growth of Pseudomonas $\mathrm{X} 2$ on decane is inhibited by decanoic acid in concentrations of $1 \mathrm{~mm}$, it is tempting to regard amide and ester formation as detoxification mechanisms.

We are indebted to Professor S. J. Pirt and Mr D. G. MacLennan for their assistance with the microbiological aspects of this work, and to Dr R. Howe for valuable discussions and provision of spectroscopic facilities. Financial support by Imperial Chemical Industries Ltd. is gratefully acknowledged.

\section{REFERENCES}

Ali Khan, M. Y., Hall, N. N. \& Robinson, D. S. (1964). Leeuwenhoek ned. Tijdschr. 30, 417.

Bergström, S., Theorell, H. \& Davide, H. (1947). Ark. Kemi Min. Geol. 23A, no. 13.

Callow, D. S. \& Pirt, S. J. (1961). J. appl. Bact. 24, 12. Cartwright, N. J. (1957). Biochem. J. 67, 663.

Jarvis, F. G. \& Johnson, M. J. (1949). J. Amer. chem. Soc. $71,4124$.

Lemieux, R. U. \& Giguere, J. (1951). Canad. J. Chem. 29, 678.

MacLennan, D. G. \& Pirt, S. J. (1967). J. gen. Microbiol. 45, 289.

Romero, E. M. \& Brenner, R. R. (1966). J. Bact. 91, 183.

Thijsse, G. J. E. \& Van der Linden, E. C. (1963). Leeuwenhoek ned. Tijdschr. $29,89$.

Van der Linden, A. C. \& Thijsse, G. J. E. (1965). Advanc. Enzymol. 27, 469.

ZoBell, C. E. (1950). Advanc. Enzymol. 10, 443. 